\title{
CONFORMIDADE DA LOCALIZAÇÃO E DIMENSÕES DA BARRA DE TRAÇÃO SEGUNDO A NORMA NBR 7811 EM TRATORES AGRÍCOLAS
}

\author{
João Adelmo da Costa de Almeida ${ }^{1}$, Airton dos Santos Alonço ${ }^{2}$, Gessieli Possebom ${ }^{1}$ \\ ${ }^{1}$ Departamento de Engenharia Rural, Universidade Federal de Santa Maria, 97105-900, Santa Maria, Brasil. \\ *E-mail: costadalmeida@hotmail.com
}

Recebido em: 07/06/2010 Aceito em: 08/01/2020

\section{RESUMO}

O crescente uso de tratores agrícolas no meio rural visa, especialmente, otimizar processos, aliado a minimização de esforços físicos. Entretanto, é necessário que estas máquinas estejam condizentes com as normativas a fim de garantir a segurança nas operações. Assim, o objetivo desse estudo foi verificar a localização e dimensões da barra de tração, conforme a norma NBR 7811, em tratores agrícolas. Foram avaliados 14 modelos de tratores agrícolas de pneus, com potência superior a 50 cv, distribuídos em quatro diferentes marcas. A coleta de dados resultou da verificação da localização e dimensões da barra de tração (NBR 7811) com o auxílio de uma trena. As dimensões denominadas " $d$ ", "b" e "C" se destacaram como as mais condizentes com a norma, com total conformidade em todos os modelos de tratores avaliados. A dimensão "A" foi de maneira geral, a variável que apresentou menores índices de conformidade, 50\% nas categorias 1 e 2 e $75 \%$ na categoria 3, caracterizando uma conformidade total de 57\%. Em relação ao nível de conformidade total comparativamente entre as categorias de classificação, os modelos de tratores analisados como tipo 3 apresentaram maior conformidade $(75 \%)$ e consequentemente os modelos de menor potência (categorias 1 e 2), resultaram em menor concordância com a norma vigente. A desconformidade parcial da norma é preocupante, uma vez que somente a partir dessa conformidade tem-se a garantia de condições ergonômicas adequadas aos operadores, ação preventiva a possíveis acidentes ocupacionais.

Palavras-chave: Ergonomia. Mecanização. Segurança.

\section{Introdução}

A evolução da agricultura está diretamente vinculada ao uso intensivo de máquinas e implementos agrícolas. A necessidade de aumento da produção de alimentos e madeira em paralelo a expansão da área dominável, levou os produtores rurais a adquirirem máquinas, visando especialmente otimização da produção agrícola, associado a minimização dos esforços físicos [1].

Dentre as máquinas agrícolas inseridas no meio rural, o trator agrícola é caracterizado como a principal fonte de energia. Com a finalidade de transportar, tracionar e fornecer potência para implementos, é amplamente utilizado em atividades agrícolas, especialmente pela elevada versatilidade de operação [2].

Entretanto, segundo Fernandes et al. [3] e Corrêa et. al., [4] o crescente uso de tratores agrícolas expõe os trabalhadores a situações de risco, tornando a atividade agrícola um dos setores que apresentam maior propensão a acidentes. Conforme Couto [5] em torno de $20 \%$ dos acidentes de trabalho na agricultura são relativos a tarefas com tratores agrícolas, sendo $54 \%$ de acidentes leves e 14,8\% dos acidentes graves causados devido à condição de equipamentos inadequados.

A severidade dessas ocorrências é apontado como preocupante, pois de acordo com os dados obtidos por Lubicky; Judy [6], na análise do índice de fraturas e amputações em crianças e adolescentes após acidentes em propriedades rurais nos EUA, o trator agrícola foi à máquina mais comumente associada a essas lesões $(33,2 \%)$. Esta informação é corroborada por Madeira [7], onde dois terços dos acidentes ocorridos em propriedades de cinco regiões diferentes de Minas Gerais, estão relacionados com tratores agrícolas, sendo o contato com as partes como TDP e barra de tração, a natureza de maior frequência dos acidentes.

Assim, a operação segura envolve fatores de ergonomia e de segurança, tanto na fabricação, quanto no uso das máquinas. Segundo Romano [8], um processo consolidado de desenvolvimento de novas máquinas deve atender a esses fatores, em conferência as normas e padronizações previstas ás maquinas agrícolas. 
A padronização dos projetos referentes aos tratores agrícolas fabricados nacionalmente está assegurado pela Associação Brasileira de Normas Técnicas (ABNT), que dentre as normas, rege considerações em relação a barra de tração, importante ponto de disponibilidade de potência dos tratores.

A barra de tração tem por função tracionar os implementos ou equipamentos de arrasto e está situada no centro do trator na altura do seu centro de gravidade. A NBR 7811 Tratores agrícolas - Características e posição da barra de tração, define a localização e as dimensões da extremidade da barra, do envolvente do garfo e dimensões das partes que constituem a barra de tração [9].

Assim, perante as especificações previstas nas legislações, o objetivo desse estudo foi verificar a localização e dimensões da barra de tração, conforme a norma NBR 7811, em tratores agrícolas.

\section{Metodologia}

\subsection{Localização e caracterização do estudo}

O estudo foi desenvolvido no município de Santa Maria, região central do estado do Rio Grande do Sul. Para o levantamento dos dados, foram selecionados tratores de diferentes modelos, os quais representam as seguintes marcas: Case, Ford, Massey Fergusson e New Holland. A escolha do local, das marcas, bem como do número de tratores avaliados foi baseada na disponibilidade de recursos (material, humano e infraestrutura).

Por ser um estudo acadêmico, sem objetivo de julgar as empresas fabricantes das marcas abrangidas, foi atribuída a denominação de Marca A, Marca B, Marca C e Marca D, para referirem-se às marcas analisadas. A delimitação do estudo objetivou avaliar as características originais de fábrica dos diferentes modelos de tratores, sem se preocupar com a ocorrência de alterações que podem ser causadas pelo usuário ou por desgaste através do uso a campo.

\subsection{Coleta de dados}

A coleta dos dados referentes a este estudo abrangeu uma amostra de 14 diferentes modelos de tratores agrícolas de pneus, com potência superior a $50 \mathrm{cv}$, sendo: a) um modelo da Marca A; b) dois modelos da Marca B; c) dois modelos da Marca $\mathrm{C}$ e, d) nove modelos da Marca D.

Para posterior análise, os tratores foram agrupados por faixas de potência do motor, segundo descrição da ABNT NBR ISO $730-2011$ [10], como segue: $1 \mathrm{~N}$ ) até $35 \mathrm{~kW}$; 1) até $48 \mathrm{~kW}$; $2 \mathrm{~N} / 2$ ) entre $30 \mathrm{~kW}$ e $92 \mathrm{~kW} ; 3 \mathrm{~N} / 3$ ) entre $60 \mathrm{~kW}$ e $185 \mathrm{~kW}$; e 4 $\mathrm{N} / 4$ ) entre $110 \mathrm{~kW}$ e $350 \mathrm{~kW}$ (Tabela 1).

Tabela 1 - Principais especificações técnicas dos tratores agrícolas avaliados.

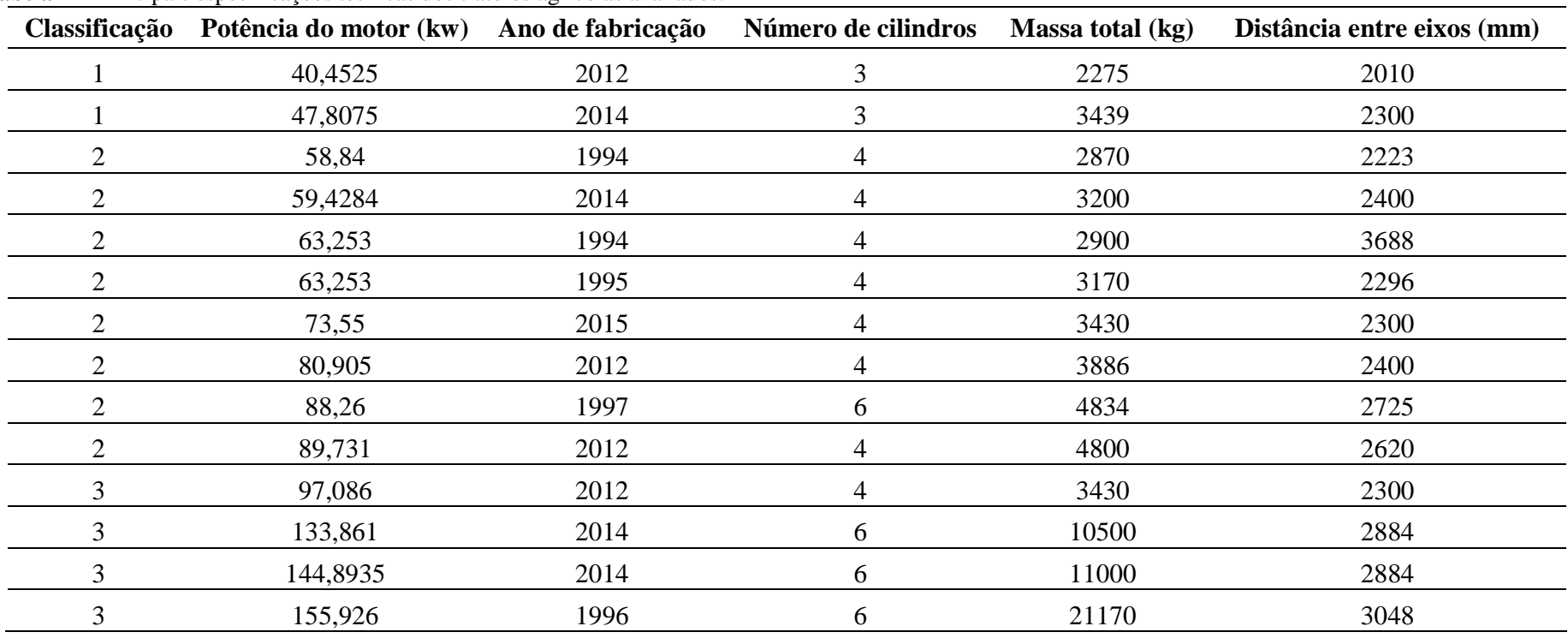




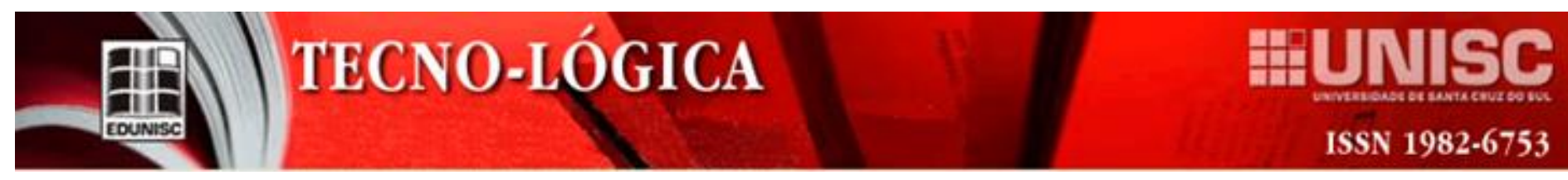

A aquisição dos dados resultou da verificação da caracterização e posição da barra de tração, segundo a NBR 7811 [9], sendo mensurados através de uma fita métrica de 3 metros, marca Tramontina Master 43156/303 (Figura 1 e Tabela 2).

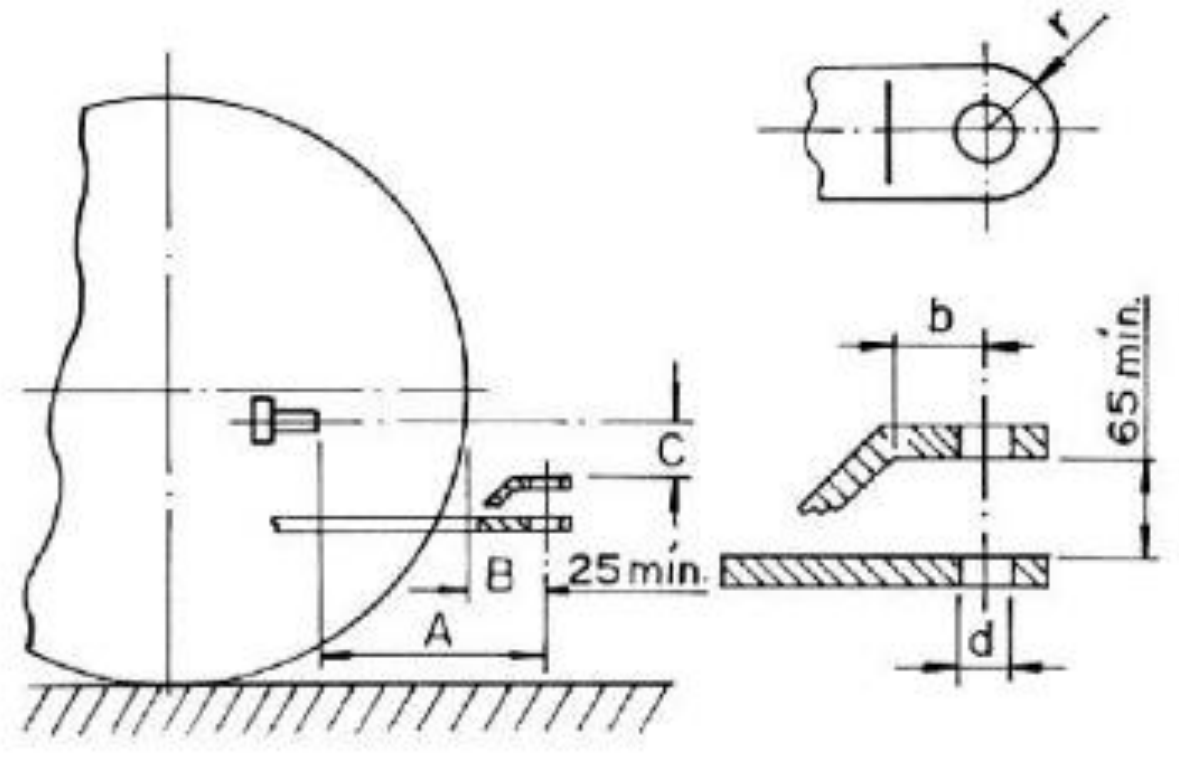

Figura 1 - Localização e dimensões da barra de tração

Tabela 2 - Dimensões e localização da barra de tração.

\begin{tabular}{cccccc}
\hline $\begin{array}{c}\text { Categoria } \\
\text { conforme } \\
\text { NBR 8566* }\end{array}$ & $\begin{array}{c}\text { Dimensões da } \\
\text { extremidade da } \\
\text { barra }(\mathrm{mm})\end{array}$ & $\begin{array}{c}\text { Envolvente } \\
\text { do garfo } \\
(\mathrm{mm})\end{array}$ & $\begin{array}{c}\text { Localização } \\
(\mathrm{mm})\end{array}$ \\
\cline { 2 - 6 } & $\mathrm{d}(+10)$ & $\mathrm{b}(\mathrm{mín})$ & $\mathrm{r}^{(\mathrm{A})}$ máx. & $\begin{array}{c}\mathrm{C}^{(\mathrm{B})} \\
(\mathrm{mín})\end{array}$ & $\begin{array}{c}\mathrm{A} \\
( \pm 10)\end{array}$ \\
\hline 1 & 33 & 60 & 70 & 200 & 400 \\
\hline 2 & 33 & 60 & 70 & 200 & 400 \\
\hline 3 & 33 & 70 & 80 & 200 & 500 \\
\hline
\end{tabular}

*substituída por ABNT NBR ISO 730 - 2011 [9]

Fonte: NBR 7811 (1993) [8].

Este procedimento foi adotado de modo análogo para cada modelo de trator disponível. Posteriormente, os dados coletados foram digitalizados em arquivo eletrônico no formato Microsoft Excel®, contendo informações sobre os modelos, potência, marca, localização e dimensões da barra de tração.

\section{Resultados e Discussão}

Do total de 14 modelos analisados neste estudo, 2 se enquadram na categoria tipo 1 , o que corresponde a $14,28 \%$ da amostra. Em relação a categoria tipo 2 e tipo 3, representadas por 8 e 4 modelos, caracterizando $57,14 \%$ e $28,57 \%$ da amostra, respectivamente. Na Figura 2 é apresentado o nível de conformidade das dimensões da barra de tração, para cada categoria, além da conformidade total.

As dimensões denominadas "d", "b" e "C" apresentaram total conformidade em todos os modelos de tratores avaliados, independente da categoria, destacando-se como as variáveis mais condizentes com a norma.

Em relação a dimensão "r", apenas a categoria tipo 1 apresentou conformidade total, entretanto, nas demais ainda se manteve consonância de $75 \%$ com a norma vigente. Entretanto, a dimensão "A" foi de maneira geral, a variável que apresentou menores índices de conformidade, $50 \%$ nas categorias 1 e 2 


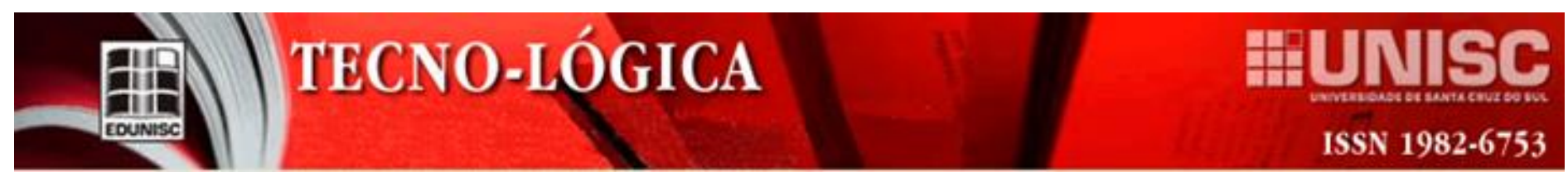

(Figura 1 A e 1 B) e 75\% na categoria 3 (Figura $1 \mathrm{C}$ ), caracterizando uma conformidade total de 57\% (Figura 1 D).

Essa última dimensão faz referência a distância horizontal entre a linha de centro do furo do pino de engate da máquina/implemento agrícola e o plano vertical que passa pela extremidade posterior da árvore da tomada de potência, de modo que a desconformidade apresentada pode permitir o acesso às partes móveis, inadvertidamente ou por imprudência, agravando os riscos a acidentes [11], como nos casos noticiados na mídia virtual [12 - 15] e na literatura técnica [16 - 18].
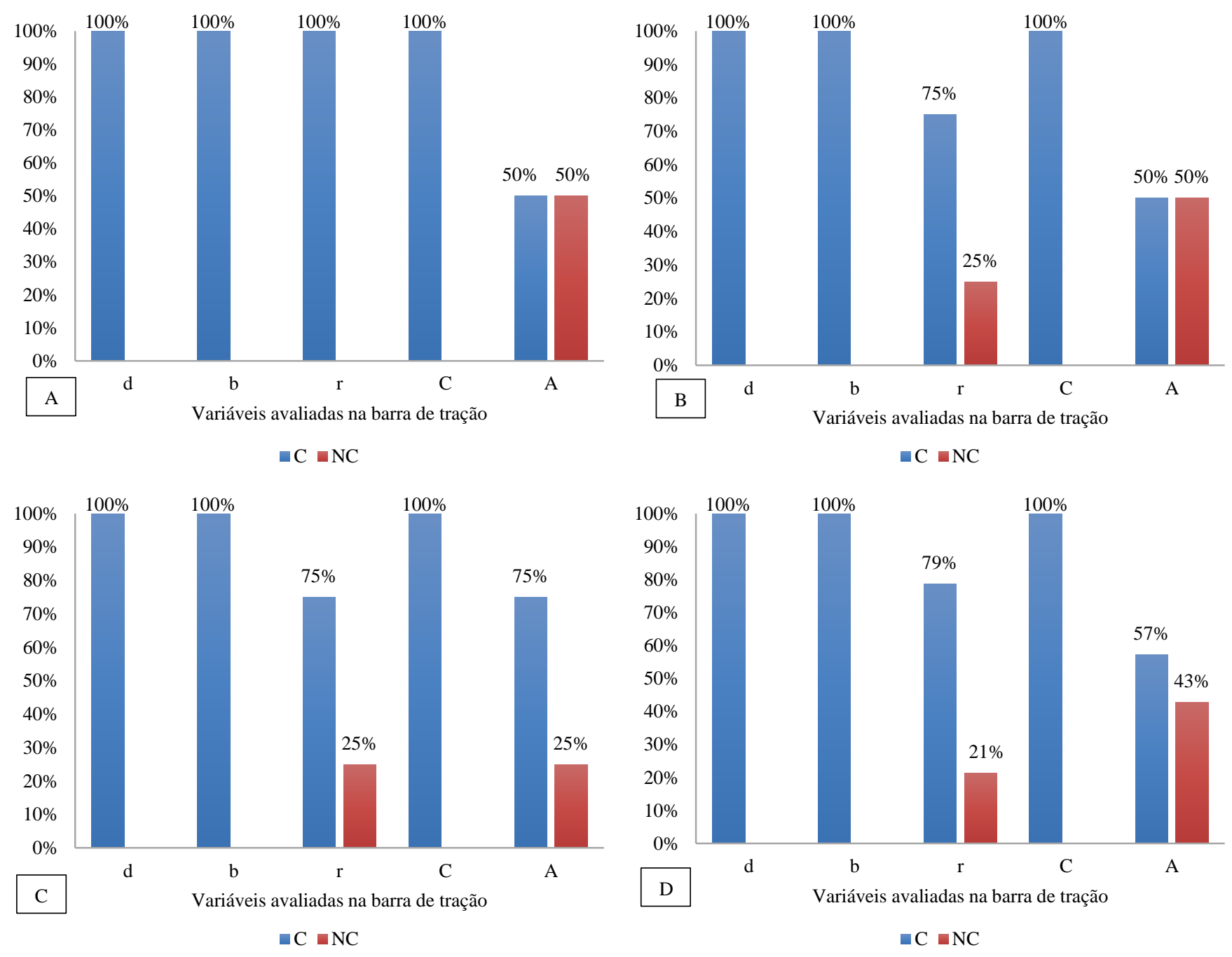

Nota: C: Conforme; NC: Não conforme

Figura 2 - Nível de conformidade das dimensões e localização da barra de tração tipo 1 (A), tipo 2 (B), tipo 3 (C) e o nível de conformidade total (D). 
Quando analisado o nível de conformidade total comparativamente entre as categorias de classificação (Figura 3), é possível observar que os modelos de tratores analisados como tipo 3 apresentaram maior conformidade (75\%). Enquanto que os modelos de menor potência (categorias 1 e 2), resultaram em menor concordância com a norma vigente. Este resultado pode ter sido influenciado pelo distinto tamanho da amostra, entre as categorias de classificação.

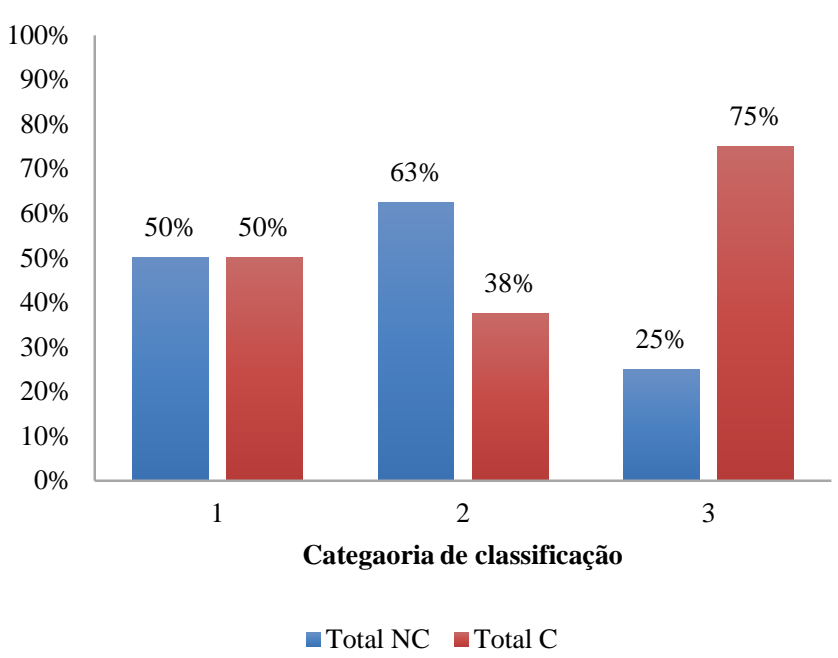

Nota: Total NC: Total Não conforme; Total C: Total conforme

Figura 3 - Nível de conformidade total segundo categoria de classificação

A total conformidade com a norma, das categorias de menor potência, é de suma importância, uma vez que segundo dados da ANFAVEA (2017) [19], nos últimos dois anos, 47\% do total das vendas de tratores foram de modelos com até $59,7 \mathrm{~kW}$ de potência e aproximadamente $30 \%$ na faixa de potência de 59,7 e $97 \mathrm{~kW}$.

Segundo Debiase; Schlosser [16], a desconformidade de uma norma pode trazer inúmeras consequências negativas. Em estudo realizado pelos autores, a falta de proteção das partes móveis do trator e implemento a ele conectado, foi uma das principais causas de $75 \%$ dos acidentes que envolveram tratores na região central do RS.

Corroborando esses resultados, Fernandes, et. al., [4], ao realizar uma caracterização dos acidentes com tratores agrícolas em algumas regiões do estado de Minas Gerais, observou que dentre as várias naturezas de acidentes encontradas, o que apresentou maior nível de risco, foi o contato com as partes móveis do trator e com o acoplamento implemento na barra de tração.
Para Lima et al. [20], máquinas são colocadas no mercado sem qualquer preocupação aparente por parte de seus fabricantes com relação a determinados parâmetros indispensáveis para a realização de trabalhos, como conforto e segurança, o que tem favorecido a ocorrência de acidentes em tratores e implementos agrícolas. Mattar et. al. [21] determinaram a necessidade de maior atenção por parte dos fabricantes nacionais de tratores agrícolas à importância da adequação às exigências de normas relacionadas à segurança ocupacional, de acesso e saída dos postos de operação de tratores. Além disso, Balestra [22] refere que as empresas fabricantes de tratores, apesar de conhecerem determinadas normas técnicas, não as utilizam, contribuindo para que os produtos não sejam padronizados.

Independente do mercado consumidor, é imprescindível que os tratores agrícolas estejam totalmente condizentes com a legislação e normas aplicáveis, a fim de reduzir as possíveis causas de acidentes. Segundo Mattar et al. [21] a conformidade com as normas, possibilita melhores condições ergonômicas aos operadores, na relação de trabalho com tratores agrícolas, agindo como ação preventiva a possíveis acidentes ocupacionais.

\section{Conclusões}

Apesar da barra de tração ser uma fonte de potência de suma importância nos tratores agrícolas, ainda é observado uma desconformidade parcial em relação a norma regulamentadora, $o$ que agrava a possibilidade de acidentes.

\section{Agradecimentos}

À propriedade que concedeu infraestrutura para realização das atividades e ao Laboratório de Pesquisa e Desenvolvimento de Máquinas Agrícolas (LASERG) pelo auxílio.

\section{CONFORMITY OF STANDARDS NBR 7811 AND NBR ISO 730 IN AGRICULTURAL TRACTORS}

ABSTRACT: The growing use of agricultural tractors in rural areas is especially aimed at optimizing processes, together with the minimization of physical effort. however, it is necessary that these machines comply with the regulations in order to guarantee safety in operations. Thus, the aim of the study was to verify the location and dimensions of the drawbar, according to norm NBR 7811, in agricultural tractors. Was evaluated 14 models of agricultural tractors of tires, with power over $50 \mathrm{hp}$, distributed in four different brands. the data collection resulted from the verification of the location and dimensions of the drawbar (NBR 7811) with the help 
of a set. The dimensions denominated "d", "b" and "C" stood out as the most consistent with the standard, with full compliance in all models of tractors evaluated. The "A" dimension was, in general, the variable with the lowest compliance rates, $50 \%$ in categories 1 and 2 and $75 \%$ in category 3 , with a total compliance of $57 \%$. Regarding the level of total compliance comparatively between classification categories, tractor models analyzed as type 3 showed higher compliance (75\%), while lower power models (categories 1 and 2), resulted in lower agreement with standard. The nonconformity of the standards is worrying, since only from this conformity is the guarantee of ergonomic conditions appropriate to the operators, preventive action to possible occupational accidents

Keywords: Drawbar. Mechanization. Safety.

\section{Referências}

[1] GERGOLETTI, I. F. (2008). Food production: A comparative analysis of scenarios in the perspective of environmental sustainability (p. 191, Doctoral dissertation, Universidade Metodista de Piracicaba, Santa Bárbara d' Oeste, SP, Brazil).

[2] RINALDI, P. C. N., FERNANDES, H. C., TEIXEIRA, M. M., CECON, P. R., \& ALVARENGA, C. B. DE. (2016). Diagnosis on the power and torque of agricultural tractors manufactured and commercialized in Brazil. Revista $\begin{array}{llll}\text { Engenharia na } & \text { Agricultura, 236-256. }\end{array}$ https://doi.org/10.13083/reveng.v24i3.644

[3] FERNANDES, H. C.; MADEIRA, N. G.; TEIXEIRA. M. M.;CECON, P.R.; LEITE, D. M. Acidentes com tratores agrícolas: natureza, causas e consequências. Engenharia na Agricultura, Viçosa. v.22 n.4; 2014.

[4] CORRÊA, I. M.; MOREIRA, C. A.; FILIPINI, S. R.; MELLO, R. da C. Avaliação de eixos cardan agrícola em campo. Applied Research \& Agrotechnology. V.9, n.2, 2016.

[5] COUTO, J.L.V. Riscos de acidentes com tratores agrícolas. Rio de Janeiro: Universidade Federal do Rio de Janeiro. Disponível em: <http://www.segurancaetrabalho.com.br/download/tratorescouto. doc>. Acesso em: 10 dez. 2008

[6] LUBICKY, J.P.; JUDY, R.F. Fractures and amputations in children and adolescents requiring hospitalization after farm equipment injuries. Fractures and amputations in children and adolescents requiring hospitalization after farm equipment injuries. Journal of pediatric orthopedics, Indiana University School of Medicine, Indianapolis, IN 46202, E.U.A. v.29, n.5, p.435-438. 2009.

[7] MADEIRA, N.G. Segurança no trabalho nas operações com tratores agrícolas em regiões de Minas Gerais. Tese de Pós-Graduação em Engenharia Agrícola, Universidade Federal de Viçosa, Viçosa, 2011.

[8] ROMANO, L. N. Modelo de referência para o processo de desenvolvimento de máquinas

agrícolas. 2003. 321 f. Tese (Doutorado em Engenharia Mecânica) - Universidade Federal de Santa Catarina, Florianópolis, 2003.

[9] ABNT- ASSOCIAÇÃO BRASILEIRA DE NORMAS TÉCNICAS. (Brasil). NBR 7811: Tratores agrícolas - Características e posição da barra de tração. 1. ed. Rio de Janeiro: ABNT, 1993. 3 p. v. 1.
[10] ABNT- ASSOCIAÇÃO BRASILEIRA DE NORMAS TÉCNICAS. (Brasil). NBR 730: Tratores agrícolas de rodas- engate traseiro de três pontos categoria $1 \mathrm{n}, 1,2 \mathrm{n}, 2,3 \mathrm{n}, 3,4 \mathrm{n}$ e 4. 1. ed. Rio de Janeiro: ABNT, 2011. 17 p. v. 1.

[11] REIS, A. V. dos; MACHADO, A. L. T. Acidentes com máquinas agrícolas: texto de referência para técnicos e extensionistas. Editora e Gráfica Universitária Pelotas, Pelotas; 2009.

[12] GUILLEN, F. Acidente com trator deixa agricultor em estado grave em Mamborê. Gazeta do Povo. Curitiba, 05.ago, 2011. Disponİvel em< http://www.gazetadopovo.com.br/vida-e-cidadania/maringa/acidentecom- tratordeixa-agricultor-em-estado-grave-em-mambore-c0usq02un6lqqeum7vuswk18u> Acesso em 02 de agosto de 2018.

[13] PATRIARCA, P. Trabalhador rural morre em acidente com trator. 18 set. 2014. JCNet-Jornal da Cidade de Bauru. Disponível em <http://www.jcnet.com.br/Regional/2014/09/trabalhador-rural-morre-emacidentecom- trator.html> Acesso em 02 de agosto de 2018.

[14] ANON. Criança de 11 anos morre em acidente de trator. PORTAL TRI. Dionísio Cerqueira, SC. 30.jul.2015. Disponível em < http://www.portaltri.com.br/1/noticias/4/geral/44868/crianca-de-11-anos-morreem-acidente-comtrator> Acesso em 02 de agosto 2018.

[15] CARLOS, L. Jovem tem pé amputado em acidente com trator. RADAR ALTO VALE. Rio do Sul, SC. 04.abr.2016. Disponível em:http://www.radaraltovale.com/noticia/antenado/jovem-tem-o-pe-amputadoem-acidentecom- trator-26094\#.V4POTPkrLIU . Acesso em 05 de julho de 2016.

[16] DEBIASI, H. SCHLOSSER, J. F. Acidentes com tratores. Cultivar Máquinas, Pelotas, RS, n. 12, p. 28-33, maio/ junho de 2002.

[17] CORRÊA, I. M, YAMASHITA, R. Y., RAMOS, H. H., FRANCO, A. V. F. Perfil dos acidentes rurais em agências do INSS de São Paulo no ano 2000. Revista Brasileira de Saúde Ocupacional, São Paulo, SP, v. 28, n.107/108, p. 39-57, 2003. http://dx.doi.org/10.1590/S0303-76572003000200005. 2003.

[18] BEER, S.R.; DEBOY, G.R. FIELD, W.E. Analysis of 151 Agricultural Driveline-Related Incidents Resulting in Fatal and Non-Fatal Injuries to U.S. Children and Adolescents Under Age 18 from 1970 through 2004. Abstract. 2007. Disponível em<http://www.ncbi.nlm.nih.gov/pubmed/17555204>. Acesso em: 02 de agosto de 2018

[19] ANFAVEA. ASSOCIAÇÃO NACIONAL DOS FABRICANTES DE VEÍCULOS AUTOMOTORES. Anuário estatístico da indústria automotiva brasileira, 2017. Disponível em: <http://www.anfavea.com.br>. Acesso em: 02 maio. 2019.

[20] LIMA, J.S.S.; SOUZA, A.P.; MACHADO, C.C.; OLIVEIRA, R.M Avaliação de alguns fatores ergonômicos nos tratores "Feller-Buncher" e "Skidder" utilizados na colheita de madeira. Revista Árvore, Viçosa, v.29, n.2, p.291-298, 2005 .

[21] MATTAR, D. M. P.; DALlMEYER, A. U.; SCHLOSSER, J. F.; DORNELLES, M. E. Conformidade de acessos e de saídas de postos de operação em tratores agrícolas segundo norma NBR/ISO 4252. Revista Engenharia Agrícola, Jaboticabal, v. 30, n. 1, p. 74-81, 2010 .

[22] BALESTRA, M. R. G. Levantamento e identificação de símbolos gráficos utilizados para caracterizar comandos e controles de tratores agrícolas. 2008. 81 p. Dissertação (Mestrado em Engenharia Agrícola)-Universidade Federal de Santa Maria, Santa Maria, 2008. 